\title{
Los caminos emprendidos por el discurso de la interculturalidad
}

\author{
Clovis Antonio Brighenti ${ }^{1}$ \\ Linda O. González Cárdenas ${ }^{2}$
}

RESUMEN: A la hora de contribuir al debate sobre la necesidad de crear e implementar políticas educativas diferenciadas destinadas a las poblaciones históricamente excluidas en América Latina resulta indispensable analizar cuales discursos las permean. En el caso de la educación indígena algunos de estos discursos se sustentan en la interculturalidad como principio fundamentador, evidenciado por ejemplo en la modificación de marcos legislativos colonialistas donde son incluidos actos normativos que afirman y reconocen derechos y pedagogías educativas propias de los pueblos indígenas, o de forma práctica en la creación de universidades y programas universitarios que se proponen romper la imposición de un conocimiento científico y monocultural. Sin embargo, varias de estas iniciativas que en la esfera educativa promulgan la interculturalidad como principal premisa han confrontado innúmeros obstáculos y limitaciones, tanto a nivel burocrático y normativo, ya que en la esfera estatal y gubernamental no son reconocidos formalmente conocimientos "no universales", así como a nivel epistémico, al continuar prevaleciendo la ciencia occidental moderna como única forma de comprender el mundo. De esta forma, en el presente trabajo pretendemos cuestionar las mencionadas limitaciones de la interculturalidad como termino norteador y sus respectivos efectos en la educación indígena, partiendo de nuestra aproximación a algunos programas educativos interculturales en algunos países de Latinoamérica que fueron destinados a poblaciones indígenas en estos países.

Palabras Clave: Interculturalidad, Educación Indígena, América Latina.

\section{Os caminhos traçados pelo discurso da interculturalidade}

RESUMO: Ao debater sobre a necessidade de criar e implementar políticas educativas diferenciadas destinadas às populações historicamente excluídas na América Latina é indispensável analisar quais discursos as permeiam. No caso da educação indígena, alguns destes discursos são sustentados na interculturalidade como principio fundamentador, evidenciado, por exemplo, na modificação de marcos legislativos colonialistas nos quais são incluídos atos normativos que afirmam e reconhecem direitos e pedagogias educativas próprias dos povos indígenas, ou, de forma prática, na criação de universidades e programas universitários que propõem quebrar a imposição de um conhecimento cientifico e monocultural. Contudo, várias destas iniciativas confrontam inúmeros obstáculos e limitações, tanto a nível burocrático como normativo, já que na esfera estatal e governamental não são reconhecidos formalmente conhecimentos ditos "não universais", assim como no campo epistêmico, ao continuar prevalecendo a ciência ocidental moderna como única forma de compreender o mundo. Desta forma, o presente artigo pretende questionar as mencionadas limitações da interculturalidade como termo nortea-

\footnotetext{
${ }^{1}$ Doctor en Historia Cultural por la Universidade Federal de Santa Catarina (UFSC) y profesor de Historia Indígena en la Universidade Federal da Integração Latino-Americana (UNILA). clovisbrighenti@hotmail.com

${ }^{2}$ Estudiante de maestría del programa de pos graduación en Antropología de la Universidade Federal do Paraná (UFPR) y Antropóloga de la Universidade Federal da Integração Latino-Americana (UNILA).Becaria de la Coordenação de Aperfeiçoamento de Pessoal de Nível Superior - CAPES.E-mail: lindagonc93@gmail.com.
} 
dor e seus respectivos efeitos na educação indígena, partindo da nossa proximidade de alguns programas educativos interculturais no Brasil e Argentina que foram destinados a populações indígenas nestes países.

Palavras-Chave: Interculturalidade, Educação Indígena, América Latina.

\section{Interculturality ways like a discourse}

ABSTRACT: When it comes to contributing to the debate on the need to create and implement differentiated educational politics for historically excluded populations in Latin America, it is indispensable to analyze which discourses sustain them. In the case of indigenous education, some of these discourses are supported by interculturality as a basis for example, in the reference modification in colonialist laws, which include normative acts that affirm and recognize indigenous educational rights and pedagogies, practice in the creation of universities and university programs that propose to break the imposition of a scientific and monocultural knowledge. However, some of these educational initiatives promulgate interculturality as the main premise confront innumerable obstacles and limitations, both at the bureaucratic and normative levels, also in the state and governmental sphere, "non-universal" knowledge is not formally recognized, as well as at the epistemic level, as modern western science continues to prevail as the only way of understanding the world. Therefore, in the present work we intend to question the mentioned limitations of interculturality as a guiding term and it's respective effects on indigenous education, starting from our proximity to some intercultural educational programs in Brazil and Argentina that were destined to indigenous populations in these countries

Keywords: Interculturality, Indigenous education, Latin American.

\section{LOS MATICES DE LA INTERCULTURALI- DAD}

En los últimos años el termino interculturalidad ha adquirido un uso masivo en varios espacios, tanto en el ámbito educativo con la creación de programas escolares y universitarios que se proponen ser diferenciados al traer otros conocimientos a los alumnos, también en la esfera de las políticas públicas como es evidenciado en normativas que promulgan la participación de poblaciones hasta ahora invisibilizadas y el respeto por sus derechos y particularidades culturales, así como también es usado en discursos sobre prácticas económicas como el turismo caracterizándose como una actividad intercultural que "proporcione integración del turista con el destino e identidad del habitante local con su ciudad, demostrando la posibilidad de encuentros in- terculturales" (FORNÉ \& JIMÉNEZ, 2015, p. 854), entre otras áreas. Lo intercultural, por tanto, ha significado de forma simple el encuentro entre varias culturas, o la interacción de personas con orígenes diferenciados en un mismo espacio.

Al mismo tiempo, al procurar reflexionar sobre los usos que se le ha otorgado a la interculturalidad esta adquiere diferentes significados, dependiendo si es pensada dentro de una dimensión teóricoepistemológica al ser tratada como concepto académico e intelectual, o proveniente de una dimensión política y de discursos que dan origen a normativas pensadas para tratar las diversidades culturales y las relaciones que son dadas entre las mismas, o la interculturalidad pensada en un ámbito práctico como las relaciones que surgen en la cotidianeidad a partir de las interacciones dadas entre culturas. Sobre este mismo 
punto el sociólogo peruano Juan Ansión (2007) menciona que la interculturalidad puede ser analizada, por un lado, como un proyecto utópico que propone el establecimiento de relaciones equitativas entre seres y epistemes, y por otro, como aquellas relaciones que son dadas en el día a día permeadas por constante conflicto. De igual forma, para Gunther Dietz y Laura Mateos (2013) es necesario distinguir entre un plano de hechos donde la interculturalidad pueda ser evidenciada en la práctica, de otro correspondiente a propuestas sociales que visan reivindicar derechos hasta ahora negados, como por ejemplo la educación, así como a propuestas políticas que propongan formas de gestionar la diferencia dentro de un Estado Nación. Así, la interculturalidad como práctica puede ser evidenciada tanto en las propias relaciones entre culturas, como en proyectos y políticas públicas que pretenden disminuir la desigualdad social existente en los niveles económico, político y social.

Como es anteriormente mencionado, la interculturalidad es empleada como discurso en la esfera política cuando la diferencia cultural se torna en objeto de atención privilegiado para el Estado, aparentemente ya no siendo considerada como un problema que debe ser eliminado con tentativas de homogeneización, como lo fue durante gran parte del siglo XX a partir de políticas de asimilación e integración, sino como una riqueza de las naciones que debía ser protegida y celebrada. En el caso de América Latina esto comienza a transcurrir a inicios de los años ochenta cuando en el ámbito educativo, por ejemplo, fueron formulados los primeros proyectos bilingüesbiculturales en México y Ecuador, pensados para convertirse en programas educativos culturalmente situados con la realidad y demandas de las poblaciones indígenas residentes en estos países (LÓPEZ, 2013, p. 135). Así mismo, de modo general la aceptación de la diferencia se vio plasmada en las reformas de varias constituciones de países latinoamericanos que a finales del siglo XX reconocieron la existencia de diversidad cultural y étnica en el interior de sus territorios. Además, en el ámbito internacional algunos instrumentos legales creados por organizaciones internacionales se tornaron en marcos de referencia para la defensa de los derechos de estas diversidades, puntualmente en relación a la presencia indígena, como la Declaración de los Derechos sobre los Pueblos Indígenas de la Organización de las Naciones Unidas - ONU, o el Convenio 169 de 1989 de la Organización Internacional del Trabajo - OIT denominado "Sobre los pueblos indígenas y tribales" donde se propone respetar, reconocer y estimular la cultura, el idioma, la organización social y la educación propia de los pueblos indígenas (OIT, 2007). Este convenio seria ratificado hasta el momento por catorce países latinoamericanos, entre los que se encuentran Argentina (2000), Bolivia (1991), Brasil (2002), Chile (2008), Colombia (1991), Costa Rica (1993), Ecuador (1998), Guatemala (1996), Honduras (1995), México (1990), Nicaragua (2010), Paraguay (1993), Perú (1994) y Venezuela (2002).

De este modo, de una postura asimilacionista adoptada por el Estado que veía en la diferencia un problema pretendiendo homogeneizar lingüística y culturalmente a los "otros no nacionales", ahora asume una orientación que trata a la diversidad cultural como un derecho que los "otros no nacionales" poseen, y como un recurso valioso que debe ser promovido a través de deter- 
minadas políticas. Sin embargo, a la hora de analizar la postura adoptada por el Estado ante la diferencia, y la acogida del discurso de la interculturalidad por él para tratar esta última, resulta fundamental considerar lo resaltado por el sociólogo colombiano Axel Rojas (2011) en relación a que aquello considerado como diferencias no emerge de forma natural y espontánea, sino, por el contrario, que son producidas contextual y relacionalmente, ya que la diferencia "es una producción social histórica, que cambia a través del tiempo en virtud de las relaciones de poder en que se definen lo pensable para la sociedad en un particular momento histórico" (ROJAS, 2011, p.28), siendo definidos así a "los otros" como diferencia en relación a "nosotros", o a un "nosotros nacionales" para el Estado.

En este mismo sentido, para Catherine Walsh (2009) las reformas constitucionales y educativas efectuadas en varios países latinoamericanos entre las décadas de ochenta y noventa, donde la diversidad cultural y lingüística pasaría a ser reconocida por el Estado respaldándose en el discurso de la interculturalidad, en realidad responderían a una lógica multicultural de capitalismo transnacional, ya que estas reformas coinciden con la divulgación de políticas neoliberales donde grandes organizaciones internacionales trazarían las estrategias globales que debían ser seguidas para la educación, derechos humanos, entre otras áreas. El discurso intercultural aquí empleado en realidad tendría un papel "funcional" a los intereses del capitalismo global que "incorpora la diferencia mientras que la neutraliza y vacía su significado efectivo" (WALSH, 2009, p. 4). Así, la incorporación de la diferencia al Estado-nación, y de modo general al sistema-mundo, no estaría influ- enciado por un interés en mejorar las condiciones de vida de las poblaciones históricamente excluidas, sino que tendría el objetivo de "administrar la diversidad ante de lo que esta visto como el peligro de la radicalización de imaginarios y agenciamiento étnicos" (WALSH, 2009, p. 8), teniendo así el control de la diferencia y su posibilidad de designación.

Dentro de una esfera académicointelectual latinoamericana también existen importantes reflexiones respecto al concepto de interculturalidad, así como al uso de este término a la hora de describir la diversidad cultural presente en este continente. Uno de los colectivos intelectuales más conocidos por realizar este tipo de debates es el grupo modernidad-colonialidad ${ }^{3}$, que se propone discutir las relaciones de poder que fueron creadas a partir de 1492, como marco histórico de la llegada de los europeos a la América, y que se materializan en el sistema capitalista moderno actual. Adicionalmente, esta red de investigadores se propone desvendar la relación entre modernidad y colonialidad que hasta ahora ha estado camuflada dentro de las ciencias sociales y su respectivo lenguaje. Por último, una de las propuestas más significativas de este grupo, donde entraría la interculturalidad como principio, es procurar estable-

\footnotetext{
${ }^{3}$ Consiste en un colectivo de pensamiento crítico latinoamericano que se tornó relevante desde los años noventa, y que funciona como una amplia red multidisciplinar, multigeneracional y multilocal, cuyos estudios y reflexiones giran en torno al cuestionamiento sobre el origen y permanencia de diferencias culturales fundadas en relaciones coloniales de poder que fundamentan las estructuras sociales vigentes hoy en día. Entre los miembros más conocidos de este grupo de investigadores se encuentran Aníbal Quijano, Walter Mignolo, Enrique Dussel, Ramón Grosfoguel, Arturo Escobar, Santiago Castro-Gómez, Catherine Walsh, Boaventura de Souza Santos, entre otros.
} 
cer otro espacio para la producción de conocimiento desde América Latina, repensando a partir de este las categorías de pensamiento colonial europeo que fueron heredadas, generando así un giro decolonial epistémico capaz de desconstruir el "mito de la modernidad" (DUSSEL, 2000, p. 49) y la colonialidad como instrumento vigente de violencia y poder.

A la hora de proponer una opción decolonial a partir de una desobediencia política y epistémica, el filósofo argentino Walter Mignolo (2008) explica que la interculturalidad también debe ser contemplada dentro de este proyecto ya que:

inter-culturalidade nos Andes é um conceito introduzido por intelectuais indígenas para reivindicar direitos epistémicos. A inter-cultura, na verdade, significa inter-epistemologia, um diálogo intenso que é o dialogo do futuro entre cosmologia não ocidental (aymara, afros, árabeislamicos, hindi, bambara, etc.) e ocidental (grego, latim, italiano, espanhol, alemão, inglês, português). (MIGNOLO, 2008, p. 30).

En este sentido, al igual que otras propuestas presentadas anteriormente, para Mignolo la interculturalidad también es considerada como una herramienta relacional, ya que procura establecer un vínculo entre cosmologías occidentales-colonialesmodernas-universales y no occidentalespluridiversas, con el fin de producir conocimientos plurales otros.

Retomando a Catherine Walsh (2009), ahora resaltando su participación dentro del colectivo modernidad-colonialidad, esta autora procura abordar el concepto de interculturalidad siempre distinguiendo su uso funcional de su uso crítico. En el primer caso, la interculturalidad de corte funcional corresponde a aquel discurso usado estatal e internacionalmente que reconoce la diversidad y diferencia dentro del Estadonación y del mundo globalizado, pero que en ningún momento pretende realizar una crítica importante a las estructuras sociales establecidas bajo una matriz colonial. Por otro lado, la interculturalidad crítica consiste en un proyecto holístico inacabado que abarca las dimensiones política, social epistémica y ética, que, a diferencia de la interculturalidad funcional, no provendría de un discurso estatal ni académico, sino de demandas presentadas por los movimientos sociales como representantes de aquella "diferencia" sometida y subalternizada. Así:

Mientras que la interculturalidad funcional asume la diversidad cultural como eje central, apuntalando su reconocimiento e inclusión dentro de la sociedad y el Estado nacionales (uni-nacionales por práctica y concepción) y dejando por fuera los dispositivos y patrones de poder institucional-estructural -las que mantienen la desigualdad-, la interculturalidad crítica parte del problema de poder, su patrón de racialización y la diferencia (colonial no simplemente cultural) que ha sido construida a función de ello. (WALSH, 2009, p. 9).

En el ámbito educativo, por ejemplo, la interculturalidad crítica no se vería representada en programas especiales que visan ofrecer una educación intercultural bilingüe diferenciada, donde son apenas incorporados saberes excluidos al interior de la estructura educativa hegemónica; por el contrario, la interculturalidad crítica se tornaría en una herramienta que permite reconocer y discutir los dispositivos de poder colonia- 
les, que han ocultado posibilidades de ser y saber fuera de la modernidad europea. De este modo, partiendo desde una perspectiva colonial, para Walsh la interculturalidad (crítica) no consiste apenas en una mera inclusión de los grupos históricamente ignorados dentro de los marcos políticos actuales, sino en una pedagogía que permita el reconocimiento del problema colonial y así el emprendimiento de acciones políticas transformadoras.

\section{INTERCULTURALIDAD EN LA EDUCACIÓN SUPERIOR INDÍGENA EN AMÉRICA LATINA}

En América Latina la interculturalidad ha sido mayormente reconocida en el campo educativo, tanto en los niveles escolar y universitario, materializada en proyectos 0 programas interculturales dirigidos a la población indígena, constituida a partir de la relación establecida como un "conflicto/negociación" (VARGAS, 2016, p. 123) entre las demandas de comunidades, representantes de movimientos sociales y académicos, y las políticas públicas formuladas por el Estado y las directrices de organizaciones supranacionales. Sin embargo, para el sociólogo peruano Luis Enrique Lopez (2009) dependiendo de cuál de estos actores sea el ejecutor y promotor de determinado programa o proyecto educativo intercultural, este último se materializaría desde y hacia direccionalidades diferentes. La primera sería "de arriba hacia abajo", producto de gestiones estatales, académicas o supranacionales, donde no se verían contempladas poblaciones indígenas. Por otro lado, la segunda direccionalidad "de abajo hacia arriba" tendría dentro de sí la agencia indígena, ya que surgiría a partir de las iniciativas de organizaciones y movilizaciones de estos pueblos. Para este mismo autor, acentuar las diferentes direccionalidades de la aplicación de la interculturalidad -de arriba hacia abajo y de abajo hacia arribapermitiría evidenciar que aquellas propuestas que surgen desde la demanda indígena, es decir desde abajo, son las que consiguen mantenerse al corresponder con la realidad de los pueblos tradicionales, y sus exigencias a nivel educativo.

Durante la mayoría del siglo XX, desde los años cuarenta hasta los años setenta, la educación indígena de procedencia estatal se encontró bajo un modelo asimilacionista "o transicional" (LOPEZ, 2009, p. 124) que tenía la finalidad de transformar a la diferencia a partir de la imposición de una identidad y lengua nacional por medio de la escuela como embajada del Estado, haciendo uso de las lenguas indígenas apenas como un instrumento para este fin. A partir del fortalecimiento de los movimientos sociales e indígenas y de su lucha por ingresar al espacio escolar y universitario como escenario legítimo de poder, esto a partir de los años setenta, primaria un modelo educativo de mantenimiento y desarrollo (LOPEZ, 2009 , p. 127), donde se pasaría a dar reconocimiento cultural y lingüístico de las poblaciones indígenas, con el mismo modelo de escuela que pretendía progresivamente incorporar a la diferencia, aun haciendo uso de la lengua indígena como instrumento de traducción para adquirir la cultura nacional. Ya a finales de los años ochenta y noventa seria oficializada la interculturalidad como modelo educativo prominente destinado a poblaciones tradicionales, siendo incorporado al contenido no apenas el carácter lingüístico sino otros saberes no occidentales relevantes culturalmente. De esta forma, para López las culturas y lenguas indígenas 
no serían vistas apenas como un medio de conversión, sino como un recurso de transformación del Estado-nación y de las relaciones hasta el momento establecidas entre estos saberes y toda la sociedad en torno.

En la actualidad, estas tensiones entre demandas sociales y políticas públicas han generado la construcción de varias propuestas interculturales en prácticamente todos los países latinoamericanos. Para comprender la magnitud de programas interculturales en la región, específicamente relacionados con la educación superior, el Observatorio de Diversidad Cultural e Interculturalidad $^{4}$ dentro del Instituto Internacional para la Educación Superior en América Latina y el Caribe, que al mismo tiempo hace parte de la Organización de las Naciones Unidas para la Educación, Ciencia y Cultura (UNESCO), ha venido realizando una serie de mapeos de experiencias e iniciativas de educación intercultural en los países de América Latina y el Caribe publicados en varios libros y artículos ${ }^{5}$, diferenciando los programas educativos con vocación intercultural que han sido creados, "desde arriba o abajo", por iniciativa estatal o pública, privada, por organizaciones indígenas, o a

\footnotetext{
${ }^{4}$ Creado en el año 2009 después de varios encuentros a nivel regional donde investigadores, militantes y políticos del área vieron la necesidad de crear un espacio que permitiera conocer los diversos contextos políticos y sociales donde se han establecido proyectos, organizaciones e instituciones dirigidas a la promoción de una educación superior intercultural. Más información sobre el Observatorio de Diversidad Cultural e Interculturalidad en Educación Superior puede ser obtenida en el siguiente enlace: http://www.iesalc.unesco.org.ve/in dex. php?option $=$ com content\&view=article\&id $=2414: \mathrm{s}$ obre-el-observatorio\&catid $=220$ :observatoriodiversidad-cultural-\&Itemid $=848$

${ }^{5}$ Todas las publicaciones correspondientes a este observatorio se encuentras disponibles aquí: http://www.ie salc.unesco.org.ve/index.php?option=com content\&vi

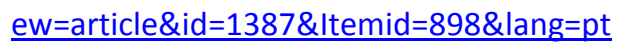

partir de convenios entre estos actores. Independientemente del origen de los programas educativos interculturales, sean ellos de procedencia pública, privada o de movimientos sociales, Daniel Mato (2008) expone que los participantes de estas iniciativas expresaron fallas en la práctica de varios programas de educación intercultural a lo largo y ancho del continente, producidas en su mayoría por falta de voluntad política, y por destinar esta modalidad exclusivamente a comunidades indígenas como un nuevo método de aculturación, desconsiderando su necesaria implementación para toda la ciudadanía.

A modo de ejemplo, resulta interesante conocer el caso de algunas experiencias de programas interculturales que se han visto seriamente obstaculizados en su práctica por diversos motivos, principalmente a la hora de ser confrontados por procesos de institucionalización, oficialización, burocratización y reconocimiento del Estado. Por ejemplo, en Ecuador la Universidad Intercultural de las Nacionalidades y Pueblos Indígenas Amawtay Wasi (UIAW) comenzó a ser pensada "desde abajo" en el año de 1996 por la Confederación de Nacionalidades Indígenas del Ecuador (Conaie), vinculada con el Instituto Científico de Culturas Indígenas, siendo tan solo reconocida en el año 2004 por el Consejo nacional de Educación Superior, órgano encargado dentro del Ministerio de Educación de este país. Desde sus inicios, esta universidad trazó como objetivo: "Cultivar nuevas relaciones societales basadas en el respeto al otro, pero con equidad. Construir un espacio académico para todas las sociedades y pueblos que conforman el actual Ecuador" (VIGIL, 2014, p. 7), así como formar estudiantes que a partir del buen vivir establecieran una rela- 
ción armónica entre la Madre Tierra y el Ser Humano. Por basarse en un carácter cuestionador y opuesto al conocimiento occidental predominante, la Universidad Intercultural de las Nacionalidades y Pueblos Indígenas Amawtay Wasi fue suspendida por el Consejo de Evaluación y Acreditación del Ecuador en el año 2013, con el argumento que esta institución intercultural no poseía buenos indicadores educativos de calidad. A pesar de esta sanción institucional por parte del gobierno ecuatoriano, la institución continúa funcionando ahora como Organización Comunitaria Pluriversidad Amawtay Wasi cuya personalidad jurídica fue registrada en ese mismo año por el Consejo de Desarrollo de las Nacionalidades y Pueblos del Ecuador (CODENPE), y cuyos recursos provienen de manos particulares.

Una situación similar también se presentó en Colombia, en este caso con la Licenciatura en Pedagogía de la Madre Tierra, creada en el año de 2008 a partir de un convenio entre la Organización Indígena de Antioquia y la Universidad de Antioquia (de carácter público), con la intención de establecer un programa universitario que enseñara contenidos acordes a la realidad de las comunidades indígenas de la región, respetando la importancia de la tierra en todas las actividades educativas. En el año 2013 se graduaron los primeros estudiantes de esta licenciatura, sin embargo, a pesar de este programa haber sido aprobado por el Ministerio de Educación en el año 2011, los diplomas de estos estudiantes no fueron certificados por el mismo ministerio ${ }^{6}$. El motivo principal era que los estudiantes

\footnotetext{
6 "Los indígenas que no podrán graduarse de la U", disponible en El Espectador aquí: https://www.elespecta dor.com/noticias/educacion/los-indigenas-no-podrangraduarse-de-u-articulo-440685
}

debían presentar un examen de conocimientos que todos los estudiantes colombianos de pregrado realizan al culminar sus estudios, cuyas preguntas se relacionan a contenidos de las típicas carreras universitarias, no abordadas por la Licenciatura en Pedagogía de la Madre Tierra. Así, al igual que la Universidad Intercultural de las Nacionalidades y Pueblos Indígenas Amawtay Wasi, esta licenciatura fue puesta en jaque por no ofrecer algunos conocimientos considerados como científicos y generales exigidos por instituciones estatales.

En otro contexto, durante aproximadamente dos años acompañamos la intención de creación de la Licenciatura Intercultural Indígena en la Universidade Federal da Integração Latino-Americana (UNILA), localizada en Foz do Iguaçu (Brasil), la cual visaba atender específicamente a las demandas educativas de la población indígena Guarani que habita en la triple frontera entre Argentina, Brasil y Paraguay. La Licenciatura en Educação Intercultural Indígena fue aprobada desde el año 2014 con previsión para iniciar en el año 2016, siendo realizados en ese intervalo diversas visitas a comunidades Guarani para registrar sus demandas y darlas a conocer a las directivas de la universidad, así como a las instituciones gubernamentales de estos tres países que podrían facilitar cuestiones burocráticas como el ingreso de estudiantes Guarani, y el reconocimiento de este curso en los tres países. A pesar de que burocráticamente esta licenciatura no tuviera aparentemente obstáculos, ya que fue aprobada su existencia y su proyecto pedagógico al interior de la UNILA, así como apoyada por instituciones gubernamentales de Argentina y Paraguay, y por las propias poblaciones Guarani de la triple frontera, en este caso las limitaciones 
eran respecto a los recursos financieros para hacer funcionar a este curso de pregrado. Desde entonces, la implementación de la Licenciatura en Educación Intercultural Indígena ha estado postergándose en dicha institución hasta conseguir el financiamiento necesario, en detrimento de otros cursos de pregrado con más apoyo institucional a los que les fue dada prioridad en los anteriores años.

A partir de estas experiencias relatadas es posible evidenciar que, independientemente de la procedencia de las iniciativas de creación de programas o proyectos interculturales, sean estas "de abajo hacia arriba" como es el caso de la Licenciatura en Pedagogía de la Madre Tierra que surgió a partir de la movilización de la Organización Indígena de Antioquía, "de arriba hacia abajo" como se tornó en algún momento la Universidad Intercultural de las Nacionalidades y Pueblos Indígenas Amawtay Wasi, o de una mediación entre iniciativas de arriba y abajo como fue el caso de la construcción y tentativa de ejecución de la Licenciatura Intercultural Indígena de la Universidade Federal da Integração Latino-Americana, en todas estas se encontraron dificultades para el desenvolvimiento de programas interculturales, en su mayoría por limitaciones burocráticas que no priorizan este tipo de proyectos educativos como posibilidades de conocimiento. De esta forma, la interculturalidad como herramienta educativa tan reconocida en el papel y en el discurso se vería "limitada por decreto", como titula Sergio Hernández-Loeza (2016) su trabajo sobre las universidades interculturales en México, que "en lugar de brindar un marco para el ejercicio de derechos de libre determinación y autonomía de los pueblos indígenas y construcción de una sociedad pluralista, se presentan como estrategias de dominación política" (HERNÁNDEZ-LOEZA, 2016, p. 114).

Una de las principales dificultades que han afrontado proyectos y programas interculturales aplicados a diversas áreas fueron señalados por Gunther Dietz en el Primer Coloquio Internacional Educación intercultural, ontologías indígenas y medicina tradicional llevado a cabo en el año 2016, quien resaltó que al intentar analizar la interculturalidad en cualquiera de sus formas de aplicación siempre aparece el Estado como principal emisor de la misma, ya que es él quien crea, acciona y define que es intercultural, o a cuál segmento de la sociedad le corresponde "ser intercultural". Como fue relatado anteriormente en el caso latinoamericano, cuando es usada la palabra intercultural inmediatamente es accionada la categoría indígena como alteridad o diferencia, desconsiderando que la interculturalidad también puede llegar a ser experimentada por otros actores sociales. Esto generaría que la interculturalidad se torne nuevamente en un instrumento colonial, al ser impuesta a los pueblos indígenas sin ser cuestionada en los grupos hegemónicos nacionales al rededor. En este mismo camino, Axel Rojas (2011) afirma que la interculturalidad y los límites que han sido evidenciados en varios programas educativos interculturales han surgido porque esta se ha convertido en un "discurso de producción de diferencias" (ROJAS, 2011, p. 105) (cursivas del autor), que acaba definiendo nuevamente las otredades como alteridades, estableciéndolos como albos de una acción intercultural proveniente del Estado, y no como una verdadera crítica a todo el sistema colonial político y social. La interculturalidad, así, no ha sido asumida como proyec- 
to de toda la sociedad en su conjunto, donde además de comunidades indígenas sea incluida la sociedad nacional, los propios Estados-nacionales, e inclusive las organizaciones supranacionales.

\section{CONSIDERACIONES FINALES}

En términos generales, la interculturalidad se ha presentado como un discurso favorable en América Latina a la hora de formular políticas educativas destinadas a poblaciones indígenas, procurando partir de la realidad lingüística, cultural y social de estas comunidades, intentando alejarse de pasadas tentativas que buscaron apenas la integración y asimilación de los indígenas a una cultura nacional definida por el Estado. A pesar de existir emprendimientos generados "desde abajo", en los que organizaciones y liderazgos indígenas se encargan de crear sus propias políticas educativas, se deparan siempre con la institucionalización y burocratización estatal. Así, resulta problemático denominar una política de intercultural cuando en realidad solo un lado de la relación la define y reconoce, en este caso el Estado-nación. Resulta así indispensable, como afirma Fidel Tubino (2007, p. 100), fomentar una "interculturalidad de doble vía" como una forma de desarrollarla no apenas como una política de tratamiento de la diversidad, sino para toda la sociedad en general. Al formular una política o proyecto intercultural reservado apenas para integrar a las culturas indígenas, se estaría reiterando las mismas pretensiones que tuvieron los proyectos integracionistas y asimilacionistas del siglo XX. De este modo, considerando la propuesta de Ansión (2007) de generar un proyecto ciudadano intercultural, es importante ir más allá de la formulación de políticas que plantean una mera inclusión, y estimular la participación efectiva de la diversidad en varios espacios de la sociedad.

Así mismo, es necesario a la hora de reflexionar en torno al concepto de interculturalidad, que esta noción dentro del movimiento indígena latinoamericano nació trascendiendo la esfera educativa, relacionando también cuestiones correspondientes a la tierra y sus derechos de acceso, así como la participación del escenario político que siempre les fue negado. Esta noción iría mucho más allá, procurando un espacio dentro de la sociedad nacional, donde sea posible cuestionar las estructuras políticas y sociales al plantear nuevos modos de conocimiento y relacionamiento. De ese modo, como afirma Catherine Walsh (2009), la interculturalidad no puede ser apenas considerada como un modelo más para definir la diferencia, o un nuevo paradigma educativo para pensar la escolarización de los pueblos indígenas, sino debe afrontarse desde su inicio como algo por construir, específicamente como proyecto político, social y epistémico que contribuya al reconocimiento de las relaciones de poder que mantienen una estructura colonial discriminatoria. Si bien no se ha conseguido el establecimiento de un dialogo equitativo entre culturas, y en el caso educativo entre epistemes, la existencia de programas y proyectos interculturales aún dentro de un sistema escolarizado y universitario ha generado grandes incomodos que han permitido cuestionar las bases de la educación occidental.

\section{BIBLIOGRAFÍA}

ANSION, Juan. La interculturalidad y los desafíos de una nueva forma de ciudadanía. 
In: ANSION, Juan; TUBINO, Fidel (Orgs.) Educar en ciudadanía intercultural: Experiencias y retos en la formación de estudiantes universitarios indígenas. Lima: Fondo Editorial de la Pontificia Universidad Católica del Perú, 2007. p. 37-62.

DIETZ, Gunther; MATEOS, Laura. El discurso intercultural ante el paradigma de la diversidad. In: AGUADO, Teresa; del OLMO, Margarita (Orgs.). Educacion intercultural: perspectivas y propuestas. Madrid: Editorial Universitaria Ramón Areces, 2009. p. 45-66. . Educación intercultural, ontologías indígenas y medicina tradicional. Revista En el Volcán Insurgente. Puebla, ano 6, n. 46, p. 18-26 nov./dez. 2016. Disponível em: http://enelvolcan.com/edicionvirtua |/enelvolcan0461116/HTML/files/assets/ba sic-html/page-1.html. Acesso em: $15 \mathrm{dez}$. 2017.

DUSSEL, Enrique. Europa, modernidad y eurocentrismo. In: LANDER, Edgardo (Org.). La colonialidad del saber, eurocentrismo y ciencias sociales: Perspectivas latinoamericanas. Buenos Aires: Consejo Latinoamericano de Ciencias Sociales - CLACSO, 2000. p. 41-53.

FORNÉ, Francesc; JIMÉNEZ, Celeste. La experiencia turística y su crítica intercultural. Turismo em análise. v. 26, n. 4, p. 843-858, dez. 2015.

HERNÁNDEZ-LOEZA, Sergio. Limitadas por decreto: Las restricciones normativas de las universidades interculturales oficiales en México. Revista del Cisen Tramas/Maepova, v. 4, n. 2, p. 95-119, 2016. LÓPEZ, Luis. Interculturalidad, educación y política en América Latina, perspectivas desde el Sur: Pistas para una investigación comprometida y dialogal. In: LÓPEZ, Luis (Org). Interculturalidad, educación y ciudadanía: perspectivas latinoamericanas. Boli- via: Plural Editores, 2009. p. $129-120$. MIGNOLO, Walter. Desobediência epistémica: A opção descolonial e o significado de identidade em política. Cadernos de Letras da UFF - Dossiê: Literatura, língua e identidade, n. 34, p. 287-324, 2008.

OIT, Organización Internacional del Trabajo. Convenio n. 169 sobre pueblos indígenas y tribales en países independientes. Lima: OIT, Oficina Regional para América Latina y el Caribe, 2007. 106 p.

ROJAS, Axel. Interculturalidad, el problema de las relaciones entre culturas. 2011. 128 f. Tesis (Maestría en Estudios Culturales) Facultad de Ciencias Sociales de la Pontificia Universidad Javeriana, Bogotá, 2011.

SORIA, Sofia. El "lado oscuro" del proyecto de interculturalidad-decolonialidad: notas críticas para una discusión. Tabula Rasa, $\mathrm{n}$. 20, p. 41-64, 2014.

TUBINO, Fidel. Las ambivalencias de las acciones afirmativas. In: ANSION, Juan; TUBINO, Fidel (Orgs.). Educar en ciudadanía intercultural: Experiencias y retos en la formación de estudiantes universitarios indígenas. Lima: Fondo Editorial de la Pontificia Universidad Católica del Perú, 2007. p. 91-110.

VARGAS, Paola. La interculturalidad imposible: Relaciones entre el proyecto educativo indígena y el estado ecuatoriano. El caso de la universidad Intercultural Amawtay Wasi. 2014. 176 f. Tesis (Maestría en Ciencias Sociales con Mención en Sociología)Facultad Latinoamericana de Ciencias Sociales Sede Ecuador, Quito, 2014. . Educación Superior Intercultural en Ecuador y México. Disputas y tendencias. Revista del Cisen Tramas/Maepova, v. 4, n. 2, p. 121-142, 2016 VIGIL, Nila. El cierre de la Universidad Amawtay Wasi y el concepto neoliberal de 
calidad educativa. Ponencia presentada en el X Foro Latinoamericano de Educación Intercultural, Migración y Vida Escolar en Puebla, México. 2014. Disponível em: https://www.academia.edu/9610458/EL Cl ERRE DE LA UNIVERSIDAD AMAWT AY WASI Y EL CONCEPTO NEOLIBERAL DE CALIDAD EDUCATIVA. Acesso em: 10 jan. 2018.

WALSH, Catherine. Interculturalidad crítica y pedagogía de-colonial: apuestas (des) de el in-surgir, re-existir y re-vivir. UMSA Revista (entre palabras), n. 3, 2009. 